\title{
RAILWAY TRAFFIC DISTURBANCE MANAGEMENT BY MEANS OF CONTROL STRATEGIES APPLIED TO OPERATIONS IN THE TRANSIT SYSTEM
}

\author{
FRANCISCO A. ORTEGA ${ }^{1}$, JUAN A. MESA ${ }^{2}$, MIGUEL A. POZO ${ }^{2} \&$ JUSTO PUERTO $^{3}$ \\ ${ }^{1}$ Higher Technical School of Architecture, Universidad de Sevilla, Spain. \\ ${ }^{2}$ Higher Technical School of Engineering, Universidad de Sevilla, Spain. \\ ${ }^{3}$ Faculty of Mathematics, Universidad de Sevilla, Spain.
}

\begin{abstract}
Railway systems in metropolitan areas support a high density of daily traffic that is exposed to different types of disturbances in the service. An interesting topic in the literature is to obtain action protocols in the presence of contingencies which can affect the system operation, avoiding the propagation of perturbation and minimizing its negative consequences.

Assume that, with a small margin of time (e.g. one day), the decision-maker of the transportation network is knowing that a part of the train fleet will become inoperative temporarily along a specific transit line and none additional vehicle will be able to restore the affected services. The decision to be taken in consequence will require to reschedule the existing services by possibly reducing the number of expeditions (line runs). This will affect travellers who regularly use the transit system to get around.

Consider that the decision-maker aims to lose the least number of passengers as a consequence of having introduced changes into the transit line. A strategy that could be applied in this context is to remove those line runs which are historically less used by travellers without affecting the remaining services. Another alternative strategy might be to reschedule the timetables of the available units, taking into account the pattern of arrivals of users to the boarding stations and the user behavior during waiting times (announced in situ).

The aim of this work consists of assessing the strategy of train rescheduling along the current transportation line when the supply must be reduced in order to reinforce the service of another line, exploited by the same public operator, which has suffered an incidence or emergency.

Keywords: disruption management, railways, timetable rescheduling.
\end{abstract}

\section{INTRODUCTION}

Planning of public transport systems includes decisions about the design of lines and service frequency that seek a balance between supply and demand in a given context and with a medium-long time horizon. The occasional failures in the operation of the system are usually not considered in this initial planning stage; therefore, a system, even properly designed, may be unavailable for unforeseen occurrences (such as accidents), or expected episodes of peak demand as a consequence of massive events.

In order to reduce the disruptive effect of these imbalances, the operator of a passenger transport system must be able to implement some control strategy to adjust schedules to traffic conditions in real time. The most commonly control strategies that are implemented for this purpose are express service (to skip stations with little flow of passengers), deadheading (to skip all stations during the returning trip), short-turning [1] (to select a portion of the fleet in order to serve, by means of short cycles, those route sections exhibiting high demand) and a combination of them [2-4].

On the other hand, if the incident happens in another transit line managed by the same operator, this decision-maker may additionally choose to reallocate its global fleet of vehicles, in 
order to help for recovering the operability of the affected line with an additional reinforcement of the rolling stock coming from other lines which were normally functioning $[5,6]$. A first review of models for the rescheduling of railway traffic timetables can be found in Ref. [7] and other more recent contribution, oriented to manage incidents, in Ref. [8].

Customers plan their journeys according to a known schedule, and they can become greatly inconvenienced if their trains do not arrive or depart at the expected time. In such cases, travellers can choose alternative means of transportation different than the usual ones. We propose in this article a methodology for implementing a redistribution of services along a transit line, which must be carried out by the operator by determining new schedules for trains within a series of feasible space-time windows, which have previously been set by the manager of the rail infrastructure. The objective consists of minimizing the loss of users, who can notice a change in the quality of service that until now they had been receiving.

In Section 2, a model of graphical representation for train schedules is introduced. The formulation of the decision model, in addition to its corresponding resolution algorithm, is presented in Section 3. A computational experience based on an application to a real case is implemented in Section 4. Finally, some conclusions are summarized in Section 5.

\section{A GEOMETRIC APPROACH TO MODEL TRAIN SCHEDULES}

Railway planners usually use running maps as graphic tools to help them in the planning process. A running map is a time-space diagram where possible crossings of trains can be observed. According to the approach developed in Ref. [9], a set of diagrams of train operations, associated to the stations along the transit line, can be built by following a common pattern of uniform mesh of time squares of length $h$. By using this geometric resource, activity of trains at each section $k$ can be represented in a comprehensive way. Inside the activity map of the $k$ th stretch/station, each point will indicate the arriving time ( $X$-coordinate) and the leaving time ( $Y$-coordinate) of a train.

In a generic $k$-station, each train is assigned to a unique point (timetable point, in the following) whose coordinates must be necessarily located in the upper triangle of the first quadrant above the straight line $Y=X+h$ (the upper sub-diagonal outlined in green).

For instance, Fig. 1 shows the data corresponding to three different trains. The first one spends in the $k$-station a time equivalent to $2 h$ in boarding and alighting passengers. Train 2

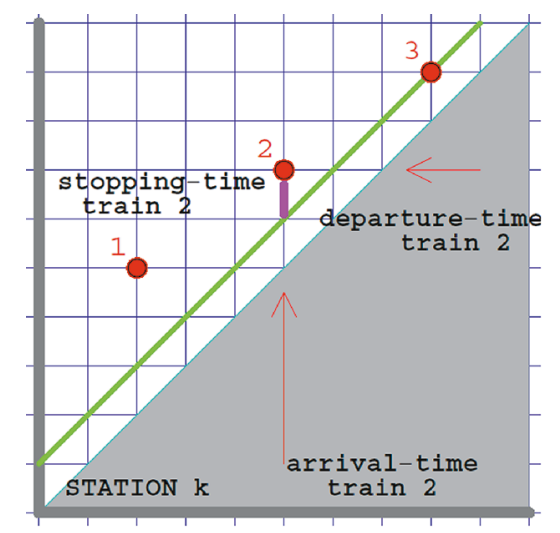

Figure 1: Three trains passing through station $k$. 
uses the minimum time required for that operation, $h$. Finally, train 3 does not stop at station $k$, and hence its position is located on the shifted diagonal in the first quadrant.

The sequence of stations (where a train may stop or not) along the railway transit line can be identified as a succession of temporary arrival-departure diagrams. As shown in Fig. 2, each timetable point in the $k$ th map will be connected to all of the feasible points of the vertical segment which starts from its projection on the diagonal inside the $(k+1)$ th temporary map.

These previous considerations lead to a directed graph structure that connects a set of feasible points belonging to arrival-departure diagrams that correspond to adjacent stations along the transit line (see Fig. 3).

Logically, the number of feasible timetable points (nodes in the graph) will be bounded by the number of tracks available into the section considered. Moreover, each timetable point in the $k$ th map will have its continuation in any of the feasible points at the vertical segment of its projection on the diagonal in the $(k+1)$ th temporary map (arcs in the graph).

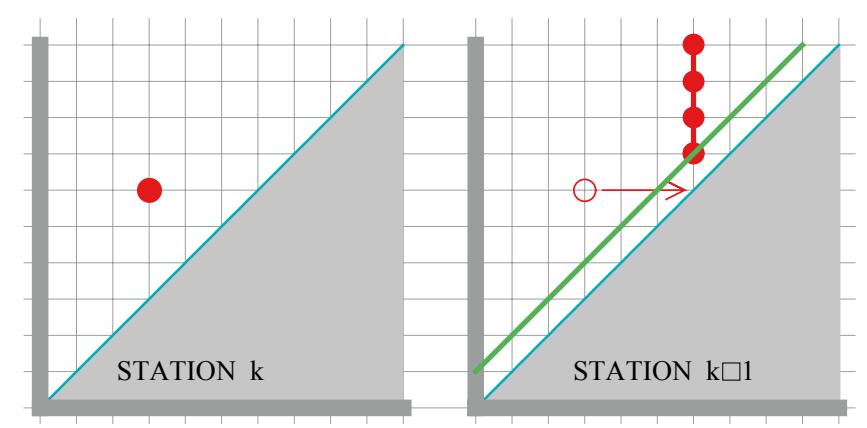

Figure 2: Feasibility zone of timetable points between consecutive stations.

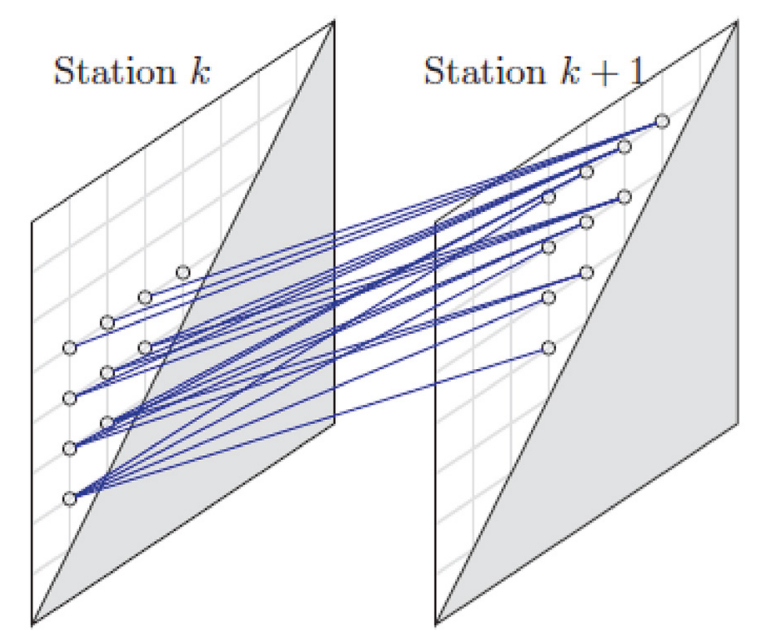

Figure 3: Arcs connecting feasible points corresponding to adjacent stations. 


\section{MODEL FORMULATION}

These considerations are included in the following formulation.

\section{Indices and sets}

$j \in J \quad$ Index identifying trains of set $J$

$k \in K \quad$ Index identifying stations of set $K$

$u, v \in T \quad$ Indexes identifying instances in the discretized time horizon $T$

$(u, v) \in M_{k} \quad$ Coordinates in a feasible temporary map $M_{k} \subset T$ at station $k$

\section{Parameters}

$A_{v}^{j k} \quad$ Population available to boarding train $j$ at station $k$ and at time $v$

\section{Variables}

$x_{u v}^{j k} \quad$ Binary variable equals to 1 if train $j$ is located at point $(u, v)$ at station $k$; 0 , otherwise

\section{Objective and constraints}

$$
z \equiv \operatorname{Max} \sum_{j \in J} \sum_{k \in K} \sum_{(u, v) \in M_{k}} A_{v}^{j k} \cdot x_{u v}^{j k}
$$

Subject to:

$$
\begin{aligned}
& \sum_{j \in J} \sum_{(u, v) \in M_{k}} x_{u v}^{j k}=|J| \quad k \in K \\
& \sum_{k \in K} \sum_{(u, v) \in M_{k}} x_{u v}^{j k} \leq|K| \quad j \in J \\
& \sum_{j \in J} \sum_{\substack{u^{\prime}<v \\
v^{\prime}>u \\
\left(u^{\prime}, v^{\prime}\right) \in M_{k}}} x_{u^{\prime} v^{\prime}}^{j k} \leq 1 \quad(u, v) \in M_{k} ; k \in K \\
& \sum_{j \in J} \sum_{\substack{u^{\prime}<v \\
v^{\prime}>u \\
\left(u^{\prime}, v^{\prime}\right) \in M_{k}}} x_{u^{\prime} v^{\prime}}^{j k} \leq n_{k}-x_{u v}^{j k} \quad(u, v) \in M_{k} ; k \in K \\
& x_{u v}^{j k} \leq \sum_{v^{\prime}>v} x_{v+1, v^{\prime} v^{\prime}}^{j, k+1} \quad(u, v) \in M_{k} ; j \in J ; k \in K-\{|K|\} \\
& x_{u v}^{j k} \in\{0,1\} \quad(u, v) \in T ; j \in J ; k \in K
\end{aligned}
$$


The objective function (1) maximizes the number of users who can be transported along the rail corridor, picking them up at their respective stations $k$ during the time interval that they are waiting on platforms, represented by set $M_{k}$. Constraints (1a) establish that the number of train schedules to be located must exactly be $|J|$. Restrictions (1b) force passage through all stations (with or without stop) for all trains to be determined. Constraints (1c) indicate that there can be no train arriving/departing from the $k$ th station if there was just another train operating. Restrictions (1e) establish that if there is an active (equals to 1) timetable point located at position $(u, v)$ of the temporary map for the $k$ th station, then there must also be another active timetable point, at the $(k+1)$ th station on the $v$ th column. Limitation of the number of trains that can operate, according to the existing number of tracks $\left(n_{k}\right)$, is indicated by means of constraints (1d). Finally, restrictions (1f) state the binary nature of the decision variables in the integer linear programming model.

In order to compute the real number of users that remains into the transit system (avoiding duplicities in the counting process), a threshold must be considered. Let $d(k, v)$ be a parameter that indicates a temporary safety distance with respect to the departure time of the last train in the initial schedule, which departed from station $k$ before time $v+1$. If such a train had not existed, then $d(k, v)=1$. Hence, once an initial schedule has been established, a value for $d(k, v)$ (greater than 1) can be easily computed, providing a security distance that avoids counting one passenger more than once.

Therefore, in order to maximize the real number of travellers kept in the system after the rescheduling, constraints (1c) can be modified as follows:

$$
\sum_{j \in J} \sum_{\substack{u^{\prime}<v \\ v^{\prime}>\max \{u, d(k, v)\} \\\left(u^{\prime}, v^{\prime}\right) \in M_{k}}} x_{u^{\prime} v^{\prime}}^{j k} \leq 1 \quad(u, v) \in M_{k} ; k \in K
$$

Additionally, an algorithm is implemented for obtaining good solutions (not necessarily optimal) within a competitive computational time. This heuristic approach can be described as follows.

\section{ALGORITHM}

[STEP 1] Generate the sequence of temporary maps corresponding to the sections of railway line. Set the mesh density of parameter $h$. Locate the existing timetable points $(u, v)$ weighted by populations $A_{v}^{j k}$.

[STEP 2] Calibrate parameter of a logit function and Estimate populations associated to the remaining not quantified timetable-points.

[STEP 3] Determine initial sets:

- Points of map $M_{k-1}$ connected to point $(u, v)$ of map $M_{k}: E_{(u, v)}^{k-}$

- Points of map $M_{k+1}$ connected to point $(u, v)$ of map $M_{k}: E_{(u, v)}^{k+}$

[STEP 4] An initial feasible graph $\mathrm{G}_{0}$ is determined by considering arc sets $E_{(u, v)}^{k-}$ and $E_{(u, v)}^{k+}$, varying from maps $k=1$ to $k=|K|-1$. Set $j=0$.

[STEP 5] While $j<|J|$

[5.1] Let index $e$ represent the arc that ends at point $(u, v)$ in the $k$ th map $M_{k}$. For each arc $e$ of graph under consideration, the weight $A_{v}^{j k}$ of its terminal node is assigned. Let $A_{e}$ denote this weight. Let $x_{e}$ be a binary variable equal to 1 if $\operatorname{arc} e$ is 
selected; 0 , in other case. By using a usual shortest-path algorithm, determine the $j$ th optimal path connecting both two terminal stations.

[5.2] According to those previously selected arcs and constraints (1a), (1b), (1c $\left.\mathrm{c}^{\prime}\right)$, $(1 \mathrm{~d}),(1 \mathrm{e})$ and (1f), redefine arc $E_{(u, v)}^{k-}$ and $E_{(u, v)}^{k+}$, giving rise to the graph $G_{j+1}$ for the next iteration.

$[5.3] j=\mathrm{j}+1$.

The geometrical model above developed can be slightly adapted in order to include the possibility of transfers between different lines within a more complex network (see Ref. [10]).

\section{COMPUTATIONAL EXPERIENCE}

As illustration, a section of the railway line with seven stations of Line C4 in the commuter train systems of Madrid (Parla [k=1] - Getafe Sector 3 [k=2] - Getafe Centro [k=3] - Las Margaritas Universidad [ $\mathrm{k}=4]$ - Villaverde Alto [k=5] - Villaverde Bajo [k=6] - Atocha $[\mathrm{k}=7]$ ) has been analysed. Table 1 shows departure times at stations of six trains that complete the itinerary Parla-Atocha in the time period [6:04, 7:00], as well as the number of passengers boarding trains in each station (data provided by the Spanish national train operator RENFE).

The time horizon [6:04, 7:00] has been split into 40 slots of size $h(1.5 \mathrm{~min})$. Table 2 shows the number of served passengers by each train.

As can readily be seen, the current fleet of six trains running according to this schedule carries 5,598 passengers. Assume that, as consequence of an incident, the system operator must reduce the fleet size by three trains. Note that if the three expeditions that carry fewer users are removed (myopic strategy), the service could only cover to 3,619 passengers, producing a loss of $35.35 \%$ of the travellers (see red at Table 2). Therefore, the subsequent strategy for train rescheduling should minimize the loss of users, by introducing advances or delays in the original schedules of the remainder three vehicles which will remain operative.

The number of passengers who remain faithful as client in the transit system, after a train supply alteration (and a possible travel time increase), can be modelled as:

Table 1: Departure timetables and passengers boarding in Line C4.

\begin{tabular}{ccccccccccccccccc}
\hline$\# j$ & \multicolumn{1}{c}{$k=1$} & \multicolumn{1}{c}{$k=2$} & \multicolumn{2}{c}{$k=3$} & \multicolumn{2}{c}{$k=4$} & \multicolumn{2}{c}{$k=5$} & \multicolumn{2}{c}{$k=6$} & \multicolumn{2}{c}{$k=7$} \\
\cline { 2 - 13 } & $t$ & Pas. & $t$ & Pas. & $t$ & Pas. & $t$ & Pas. & $t$ & Pas. & $t$ & Pas. & $t$ & Pas. \\
\hline 1 & $6: 04$ & 335 & $6: 10$ & 1 & $6: 13$ & 44 & $6: 15$ & 7 & $6: 18$ & 44 & $6: 21$ & 46 & $6: 31$ & 147 \\
2 & $6: 12$ & 177 & $6: 18$ & 5 & $6: 21$ & 113 & $6: 24$ & 48 & $6: 26$ & 124 & $6: 29$ & 81 & $6: 38$ & 302 \\
3 & $6: 16$ & 307 & $6: 22$ & 1 & $6: 25$ & 35 & $6: 28$ & 29 & $6: 30$ & 64 & $6: 34$ & 58 & $6: 42$ & 123 \\
4 & $6: 22$ & 55 & $6: 28$ & 8 & $6: 31$ & 138 & $6: 33$ & 54 & $6: 36$ & 163 & $6: 39$ & 86 & $6: 48$ & 234 \\
5 & $6: 28$ & 429 & $6: 34$ & 10 & $6: 36$ & 145 & $6: 39$ & 62 & $6: 42$ & 173 & $6: 44$ & 119 & $6: 54$ & 349 \\
6 & $6: 34$ & 511 & $6: 40$ & 4 & $6: 42$ & 102 & $6: 44$ & 26 & $6: 46$ & 153 & $6: 50$ & 115 & $7: 00$ & 571 \\
\hline
\end{tabular}


Table 2: Passengers transported by the trains (punctual arrivals at stations).

\begin{tabular}{ccccccccc}
\hline Train & Pass. 1 & Pass. 2 & Pass. 3 & Pass. 4 & Pass. 5 & Pass. 6 & Pass. 7 & Total pass. \\
\hline 1 & 335 & 1 & 44 & 7 & 44 & 46 & 147 & 624 \\
2 & 177 & 5 & 113 & 48 & 124 & 81 & 302 & 850 \\
3 & 307 & 1 & 35 & 29 & 64 & 58 & 123 & 617 \\
4 & 55 & 8 & 138 & 54 & 163 & 86 & 234 & 738 \\
5 & 429 & 10 & 145 & 62 & 173 & 119 & 349 & 1,287 \\
6 & 511 & 4 & 102 & 26 & 153 & 115 & 571 & 1,482 \\
\hline
\end{tabular}

- A two-level decision model, known as hierarchical logit model (see Ref. [11]), if the user knows the news of the rescheduling when he/she is already in the system.

- A decision model of a single level, if the user previously knows the existence of the rescheduling in advance, and its impact on the increment of travel time (decreasing the perceived utility), choosing the more convenient alternative means of transport between the available options.

The situation we assume is the second one. Since departure times of trains at stations were previously known by users, a population of potential travellers is mobilized towards the station platform, converging in time with the timely arrival of such train. Next, since users are unaware of the incident, users will gradually reach the station platforms, hoping to find on time their trains (see Fig. 4). Then, they are notified that a service rescheduling has been done and some trains can suffer delays or cancellations. This behaviour can produce a decrement in the number of passengers after rescheduling.

There are numerous models devoted to simulate the behaviour of passengers on rail/metro platforms (see, for instance, D'Acierno et al. [12]). In Ref. [9] an estimation of the number of travellers at the station platform is carried out by means of applying Taylor's formula to the logit term for the waiting-time variable. This approach produces a simple coefficient (less

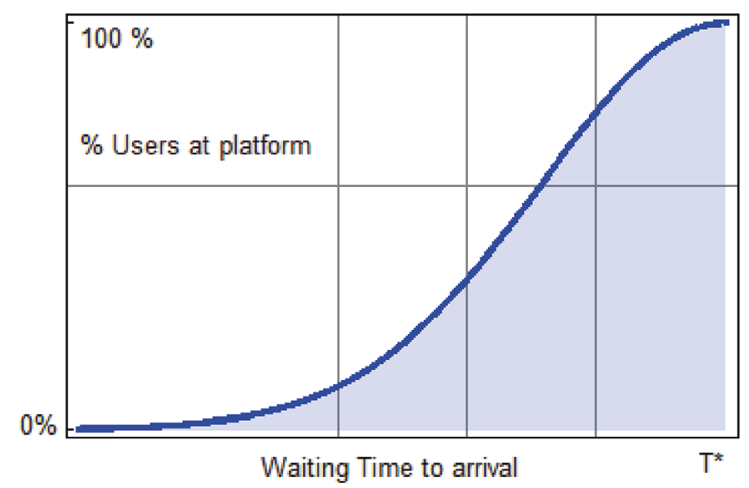

Figure 4: Percentage of users that reach the station platform. 
than 1) that, after multiplying by initial population, reduces the number of potential travellers waiting at the station platform.

Additionally, the model of demand must describe the interactions between successive waves of user arrivals to the platforms. If a train does not arrive at the scheduled time, a part of the population will leave the station while another portion would remain waiting for the next train, mixing with the next wave of users (see Fig. 5). We assume that no user waits beyond two tardy train arrivals.

As example, Fig. 6 shows the time slots and the number of passengers boarding the first three trains of the system in the first three stations of Table 1.

Given the gradual access of users to the platforms before the arrival of the train and the decline of this population, after verifying that the expected train will not circulate, we can estimate the number of potential travellers who stay at all time slots in the station platforms. The number of users, ready at platform of station 1 to board a train corresponding to the first three waves, is shown in Fig. 7 for successive time slots.

By accumulating the travellers corresponding to independent waves that meet for the same time slot on the platform, we can estimate the efficiency of new train schedules by using the before described algorithm.

Table 3 shows the three most efficient train schedules along the transit line, by assuming that every train must stop at all stations. This context is modelled by imposing equality at constraints (1b). The total number of passengers, transported by these expeditions which were determined by applying our model, is 5,010, a sensitive improvement with respect to the

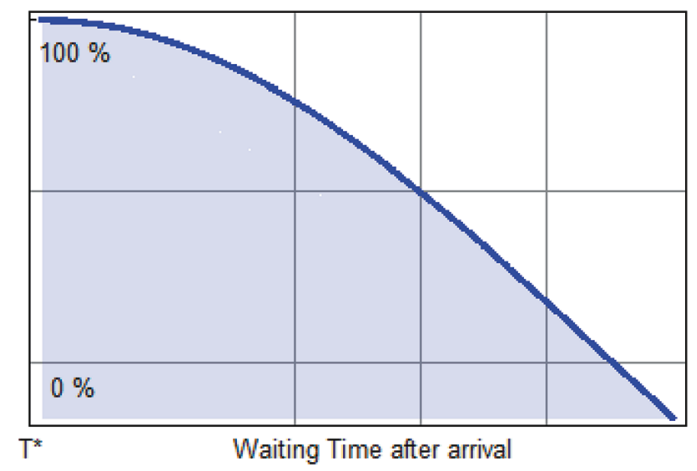

Figure 5: Percentage of users waiting at platform after expected arrival at $T^{*}$.

\begin{tabular}{|c|c|c|c|c|c|c|c|c|c|c|c|c|c|c|c|c|}
\hline TIMING & 1 & 2 & 3 & 4 & 5 & 6 & 7 & 8 & 9 & 10 & 11 & 12 & 13 & 14 & 15 & 16 \\
\hline ST1 & 335 & & & & 177 & & 307 & & & 55 & & & 429 & & \\
\hline ST2 & & & & 1 & & & & $>$ & 5 & & 1 & & & 8 & \\
\hline ST3 & & & & & & 44 & & & & & 113 & & 35 & & \\
\hline$\ldots$ & & & & & & & & & & $>$ & 7 & & & & \\
\hline
\end{tabular}

Figure 6: Number of passengers boarding trains along the transit lines. 


\begin{tabular}{|c|c|c|c|c|c|c|c|c|c|c|c|c|c|}
\hline ST1 & 1 & 2 & 3 & 4 & 5 & 6 & 7 & 8 & 9 & 10 & 11 & 12 & 13 \\
\hline Wave 1 & 335 & 314 & 295 & 277 & 260 & 244 & & & & & & & \\
\hline Wave 2 & 109 & 123 & 139 & 157 & 177 & 166 & 156 & & & & & \\
\hline Wave 3 & & & & & & 273 & 307 & 288 & 270 & 253 & 113 & \\
\hline$\ldots$ & & & & & & & & & & & & $\ldots$ & \\
\hline
\end{tabular}

Figure 7: Number of travellers ready to board the train in separate waves.

Table 3: Three most efficient train schedules along the transit line (with mandatory stops in all stations).

\begin{tabular}{|l|c|c|c|c|c|c|c|c|c|c|c|c|c|c|c|c|c|}
\hline $\begin{array}{l}\text { TIM- } \\
\text { ING }\end{array}$ & $\mathbf{5}$ & $\mathbf{9}$ & $\mathbf{1 1}$ & $\mathbf{1 5}$ & $\mathbf{1 6}$ & $\mathbf{1 7}$ & $\mathbf{2 0}$ & $\mathbf{2 1}$ & $\mathbf{2 2}$ & $\mathbf{2 3}$ & $\mathbf{2 6}$ & $\mathbf{2 8}$ & $\mathbf{2 9}$ & $\mathbf{3 2}$ & $\mathbf{3 4}$ & $\mathbf{3 5}$ & $\mathbf{4 0}$ \\
\hline ST1 & $\mathbf{4 1 7}$ & & $\mathbf{3 0 8}$ & & & $\mathbf{8 6 6}$ & & & & & & & & & & & \\
\hline ST2 & & 4 & & 8 & & & & $\mathbf{1 1}$ & & & & & & & & & \\
\hline ST3 & & & 132 & & & $\mathbf{1 6}$ & & & & $\mathbf{2 2 1}$ & & & & & & & \\
\hline ST4 & & & & & 45 & & & & 77 & & & $\mathbf{7 6}$ & & & & & \\
\hline ST5 & & & & & & 142 & & & & 215 & & & $\mathbf{2 9 5}$ & & & \\
\hline ST6 & & & & & & & & & & 106 & & & $\mathbf{1 3 2}$ & & & $\mathbf{2 1 2}$ & \\
\hline ST7 & & & & & & & & & & & & & $\mathbf{3 8 2}$ & & 335 & & 860 \\
\hline
\end{tabular}

3,619 passengers covered by the three most efficient original timetables (myopic strategy). In terms of loss of travellers, this amount only would reach a $10.50 \%$, instead of the earlier $35.35 \%$.

Following on, Table 4 shows the three most efficient line runs by assuming that not all the stations had to be visited. When a train does not stop at a station, it is possible to reduce the running time and to visit the following station at precedent time slot by capturing a possible higher number of passengers. This strategy can be modelled by imposing inequality

Table 4: Three most efficient train schedules along the transit line (without mandatory stops in all stations).

\begin{tabular}{|c|c|c|c|c|c|c|c|c|c|c|c|c|c|c|c|c|c|c|}
\hline TIMING & $\mathbf{5}$ & $\mathbf{9}$ & $\mathbf{1 1}$ & $\mathbf{1 2}$ & $\mathbf{1 5}$ & $\mathbf{1 6}$ & $\mathbf{1 7}$ & $\mathbf{2 0}$ & $\mathbf{2 1}$ & $\mathbf{2 2}$ & $\mathbf{2 3}$ & $\mathbf{2 6}$ & $\mathbf{2 8}$ & $\mathbf{2 9}$ & $\mathbf{3 2}$ & $\mathbf{3 4}$ & $\mathbf{3 5}$ & $\mathbf{4 0}$ - \\
\hline ST1 & 417 & & $\mathbf{3 0 8}$ & $\mathbf{3 9 1}$ & & & $\mathbf{8 6 6}$ & & & & & & & & & & & \\
\hline ST2 & & $\mathbf{4}$ & & & $\mathbf{8}$ & & & & $\mathbf{1 1}$ & & & & & & & & & \\
\hline ST3 & & & 132 & & & & $\mathbf{1 6 6}$ & & & & $\mathbf{2 2 1}$ & & & & & & & \\
\hline ST4 & & & & & & 45 & & & & 77 & & & $\mathbf{7 6}$ & & & & & \\
\hline ST5 & & & & & & & & 142 & & & & 215 & & & 295 & & & \\
\hline ST6 & & & & & & & & & & & 106 & & & 132 & & & $\mathbf{2 1 2}$ & \\
\hline ST7 & & & & & & & & & & & & & & $\mathbf{3 8 2}$ & & 335 & & 860 \\
\hline
\end{tabular}


at constraints (1b) in our model. Since station 2 (with a very low demand) is not visited by the second train (green sequence), it is possible to change its transit timetable for picking up more passengers at station 1 by means a later departure. By using this strategy, the number of travellers is now 5085, and the passenger loss with respect to the scenario of incidence absence is only $9.16 \%$.

\section{CONCLUSIONS}

We have presented a new approach for implementing a redistribution of service along a railway line, which must be carried out by the operator by determining new schedules for trains. Planar grids represent the basis of a geometric model for identifying possible locations for train timetables. An optimization model has been formulated in order to decide an effective rescheduling motivated by a forced reduction in the existing fleet size. This model determines in two possible scenarios (with/without mandatory stops in all stations) the more efficient solutions that can help to make operative decisions in a railway transportation system.

\section{ACKNOWLEDGEMENTS}

This research has been partially supported by the Spanish projects MTM2013-46962-C02-01, MTM2015-67706-P, and MTM2016-74983-C2-1-R (MINECO/FEDER,UE).

\section{REFERENCES}

[1] O’Dell, S. \& Wilson, N., Optimal real-time control strategies for rail transit operations during disruptions. Computer-Aided Transit Scheduling, Vol. 471, ed. N. Wilson, Springer-Verlag, Berlin, Heidelberg, pp. 299-323, 1999. DOI: 10.1007/978-3-64285970-0_15.

[2] Eberlein, X.-J., Wilson, N. \& Bernstein, D., Modeling real-time control strategies in public transit operations. Computer-Aided Transit Scheduling, Vol. 471, ed. N. Wilson, Springer-Verlag, Berlin, Heidelberg, pp. 325-346, 1999. DOI: 10.1007/978-3-64285970-0_16.

[3] Mesa, J.A., Ortega, F.A. \& Pozo, M.A., Effective allocation of fleet frequencies by reducing intermediate stops and short turning in transit systems. Lecture Notes in Computer Science, 5868(8), pp. 293-309, 2009. DOI: 10.1007/978-3-642-05465-5_12.

[4] Cortés, C.E., Jara-Díaz, S. \& Tirachini, A., Integrating short turning and deadheading in the optimization of transit services. Transportation Research Part A, 45, pp. 419-434, 2011. DOI: $10.1016 /$ j.tra.2011.02.002.

[5] Wilson, N.H.M., Macchi, R.A., Fellows, R.E. \& Decko, A.A., Improving service on the MBTA green line through better operations control. Transportation Research Record, 1361, pp. 296-304, 1992. DOI: http://onlinepubs.trb.org/Onlinepubs/ trr/1992/1361/1361-042.pdf.

[6] Soeldner, D., A comparison of control options on the MBTA green line. Master's thesis, Civil Engineering, MIT, 1993.

[7] Cordeau, J.-F., Toth, P. \& Vigo, D., A survey of optimization models for train routing and scheduling. Transportation Science, 32(4), pp. 380-404, 1998. DOI: 10.1287/ trsc.32.4.380.

[8] Törnquist, J., Railway traffic disturbance management - an experimental analysis of disturbance complexity, management objectives and limitations in planning horizon. Transportation Research Part A: Policy and Practice, 41(3), pp. 249-266, 2007. DOI: 10.1016/j.tra.2006.05.003. 
[9] Mesa, J.A., Ortega, F.A. \& Pozo, M.A., A geometric model for an effective rescheduling after reducing service in public transportation systems. Computers \& Operations Research, 40(3), pp. 737-746, 2013. DOI: 10.1016/j.cor.2011.12.003.

[10] Mesa, J.A., Ortega, F.A., Pozo, M.A. \& Puerto, J., Rescheduling railway timetables in presence of passenger transfers between lines within a transportation network. Advances in Intelligent Systems and Computing, 262, pp. 347-360, 2014. Computer-based Modelling and Optimization in Transportation, ed. J.F. de Sousa \& R. Rossi, Springer International Publishing Switzerland. DOI: 10.1007/978-3-319-04630-3_26.

[11] Daly, A., Estimating logit models. Transportation Research Part B: Methodological, 21(4), pp. 251-267, 1987. DOI: 10.1016/0191-2615(87)90026-9.

[12] D'Acierno, L., Botte, M. \& Montella, B., Assumptions and simulation of passenger behaviour on rail platforms. International Journal of Transport Development and Integration, 2(2), pp. 123-135, 2018. DOI: 10.2495/TDI-V2-N2-123-135. 